\title{
Gestão do conhecimento para o planejamento estratégico na pandemia: um estudo de caso em um observatorio de cooperativas
}

Knowledge management to strategic in pandemic: a case study in a cooperative observatory

\author{
Ieda Pelógia Martins Damian \\ Universidade de São Paulo. Faculdade de Filosofia, \\ Ciências e Letras, Brasil \\ iedapm@usp.br \\ (D) https://orcid.org/0000-0001-5364-3243
}

\section{Beatriz Rosa Pinheiro dos Santos}

Universidade Estadual Paulista "Túlio de Mesquita Filho".

Faculdade de Filosofia e Ciências, Brasil

pinheiro.santos@unesp.br

(D) https://orcid.org/0000-0001-8620-2612

\section{Nidelson Teixeira Falcão}

Universidade de São Paulo. Faculdade de Economia,

Administração e Contabilidade, Brasil

ntfalcao@hotmail.com

(iD https://orcid.org/0000-0001-9172-7085

\author{
Davi Rogério de Moura Costa \\ Universidade de São Paulo. Faculdade de Economia, \\ Administração e Contabilidade, Brasil \\ drmouracosta@usp.br \\ D https://orcid.org/0000-0001-5653-478X
}

\section{Resumo:}

O conhecimento tem ocupado posições de destaque nas organizações por possibilitar a construção de vantagens competitivas sustentáveis. Diante da importância deste recurso, sua gestão passa a ser fundamental, uma vez que contribui para que os objetivos organizacionais sejam alcançados. Estes objetivos são definidos durante a execução das atividades do planejamento estratégico, que se propõe a direcionar as organizações para que resultados pretendidos sejam alcançados. Estas atividades se fazem ainda mais importantes em momentos de crise, como os vivivos pela pandemia do COVID-19. Foi neste cenário pandêmico que o Observatório de Cooperativas da Universidade de São Paulo, por meio da utilização de tecnologias de informação e comunicação, desenvolveu seu planejamento estratégico. Perante ao exposto, este estudo tem por objetivo destacar as contribuições da gestão do conhecimento para o planejamento estratégico, uma vez que tais ferramentas se tornam ainda mais essenciais em momentos de crise como os vividos em uma pandemia. Como procedimentos metodológicos, se fez uso do estudo de caso que permite, além de relacionar teoria e pratica, demonstrar o contexto do observatório onde as atividades foram desenvolvidas. Como resultados, se destaca que as contribuições advindas da gestão do conhecimento, como o desenvolvimento de um ambiente colaborativo, do trabalho em equipe, da motivação, da autonomia, entre outros, foram essenciais para o desenvolvimento do planejamento estratégico do Observatório em meio a pandemia do COVID-19.

Palavras-chave: Gestão do conhecimento, Planejamento estratégico, Covid-19, Pandemia, Observatório de cooperativas, Brasil. 


\section{Abstract:}

Knowledge has occupied prominent positions in organizations for enabling the construction of sustainable competitive advantages. In view of the importance of this resource, its management becomes fundamental, since it contributes to the achievement of organizational objectives. These objectives are defined during the execution of the activities of strategic planning, which proposes to direct organizations so that the desired results are achieved. These activities are even more important in times of crisis, such as those that were caused by the COVID-19 pandemic. It was in this pandemic scenario that the University of São Paulo's Cooperative Observatory, through the use of information and communication technologies, developed its strategic planning. In view of the above, this study aims to highlight the contributions of knowledge management to strategic planning, since such tools become even more essential in times of crisis such as those experienced in a pandemic. As methodological procedures, a case study was used that allows, in addition to relating theory and practice, to demonstrate the context of the observatory where the activities were carried out. As a result, it is highlighted that the contributions from knowledge management, such as the development of a collaborative environment, teamwork, motivation, autonomy, among others, were essential for the development of the Observatory's strategic planning in the midst of the pandemic. of COVID-19.

KEYWORDS: Knowledge management, Strategic planning, Covid-19, Pandemic, Observatory of cooperatives, Brasil.

\section{INTRODUÇÃo}

Globalização, avanços tecnológicos e explosão informacional são importantes elementos que contribuem para a construção de um ambiente altamente competitivo, onde as mudanças são constantes e ocorrem em uma velocidade nunca antes vista. Para atuarem e se destacarem neste ambiente, as organizações precisam fazer o uso eficiente de seus recursos estratégicos. No passado, a posição de recurso estratégico organizacional era ocupada por recursos tangíveis como matéria-prima e maquinários. Atualmente, esta posição passa a ser ocupada por recursos intangíveis, como o conhecimento, pois representa o único recurso capaz de auxiliar as organizações no desenvolvimento de vantagens competitivas sustentáveis.

Diante deste cenário, é imprescindível que as organizações, sejam elas públicas ou privadas, grandes ou pequenas, físicas ou virtuais, façam a gestão adequada de seu conhecimento. Apesar de sua posição de destaque, ainda é muito comum que as organizações percam parcelas significativas de conhecimento. Pois, "apesar do conhecimento ser reconhecido como recurso estratégico em um contexto repleto de mudanças e desafios, as organizações ainda encontram muitas dificuldades para administrá-lo" (Damian, Moro-Cabero, 2020c, p. 1).

A gestão do conhecimento (GC) envolve a identificação do conhecimento que a organização precisa, as formas de obtenção deste conhecimento, meios de armazenar este conhecimento para que ele possa ser disseminado, compartilhado com todos que possam fazer uso dele sempre que necessário e, assim, gerar novos conhecimentos organizacionais e contribuir para as que as organizações alcancem seus objetivos.

Para que as organizações possam usufruir dos benefícios advindos da GC, é fator crítico de sucesso que tal gestão esteja alinhada ao planejamento estratégico organizacional.

Por fazer parte de um ambiente dinâmico, onde as mudanças são frequentes em âmbito econômico, social, tecnológico e político, as organizações, de acordo com Alday (2000), passaram a focar suas atenções no planejamento estratégico $(\mathrm{PE})$, que se concentra nas medidas positivas que uma organização pode tomar para aproveitar as oportunidades e enfrentar ameaças encontradas neste ambiente.

O PE é de fundamental importância para as organizações porque permite, entre outras coisas, definir o que se pretende no futuro, quais objetivos se almeja, quais são os resultados desejados, bem como identificar as ações e recursos necessários para que tais objetivos e resultados sejam alcançados. Em todo este processo, o conhecimento tem papel de destaque e representa um grande diferencial a ser explorado.

O alinhamento das ações de GC com as estratégias e objetivos organizacionais é, segundo Fresneda, Gonçalves, Papa e Fonseca (2009), um dos fatores que devem ser observados para que as organizações possam usufruir dos benefícios advindos de tal gestão.

A estratégia disponibiliza a organização o fundamento de como suas capacidades e recursos devem ser desenvolvidos para que os objetivos da GC possam ser alcançados (Wong, 2005). 
Mediante a importância do alinhamento entre PE e GC, este estudo se propõe a elencar as contribuições da GC no desenvolvimento do PE do Observatório de Cooperativas (ObsCoop) da Universidade de São Paulo (USP). Para tanto, além de um levantamento bibliográfico acerca dos conceitos envolvidas neste estudo, quais sejam PE e GC, apresenta-se, por meio da realização de estudo de caso, como se deu o desenvolvimento do PE do ObsCoop, realizado de modo virtual devido as imposições trazidas pela pandemia do COVID-19 e se destaca o papel da GC neste processo, bem como nos planos de ação.

O desenvolvimento deste estudo, que enfoca as contribuições da GC, encontra respaldo na seguinte afirmação:

Por se tratar de um recurso de fundamental importância para a criação de vantagens competitivas sustentáveis para as organizações, o conhecimento e sua gestão, representam um campo onde pesquisas e estudos devem ser desenvolvidos e aprofundados de maneira contínua, de modo a gerar benefícios a todos os agentes implicados como organizações, funcionários, colaboradores, parceiros, clientes, governos e cidadãos (Damian \& Moro-Cabero, 2020b, p. 1).

Além disso, "diante da posição estratégica ocupada pelo conhecimento, sua gestão se torna imprescindível para todas as organizações que desejam se manterem e se destacarem em um ambiente de grande competitividade e de mudanças constantes" (Damian y Moro-Cabero, 2020a, p. 17).

\section{Planejamento estratégico}

O mercado altamente competitivo e dinâmico faz com que as organizações busquem por ferramentas e metodologias que facilitam a prospecção de metas organizacionais para a obtenção de melhores resultados, como é o caso do planejamento, que oferece um meio de adaptação às rápidas mudanças sofridas nas últimas décadas, o que o torna parte indispensável para o alcance dos objetivos organizacionais (Cruz, Neris, VilasBoas, y Menezes, 2017).

O planejamento é um conjunto de funções administrativas que visa, através da previsão, determinar antecipadamente os objetivos a serem atingidos pelos sujeitos organizacionais, assim como o movimento processual a ser seguido para que tais objetivos sejam alcançados, segundo Chiavenato (2014).

Almeida (2010) reforça que o planejamento faz com que as organizações definam o que fazer, quando, onde e como as tarefas serão realizadas, além dos recursos necessários para atingir seus objetivos e se preparar para situações futuras, de modo que desenvolvam uma postura proativa, eficiente e eficaz, que contribuirá para o alcance das metas organizacionais. Se destaca que, atualmente, o recurso estratégico das organizações é o conhecimento, porquanto sua gestão deve fazer parte do planejamento organizacional.

Para Cruz et al. (2017) o planejamento é uma necessidade básica e comum para todas as organizações que buscam se estabelecer de maneira positiva e com diferenciais competitivos no mercado. Todo planejamento exige o levantamento e a elucidação dos pontos fortes e fracos, a fim de potencializá-los e corrigi-los, respectivamente. Por ser constituir de elementos como valores e experiências, o conhecimento é um recurso intangível de dificil imitação, de modo que sua gestão contribui para a construção de vantagens competitivas sustentáveis, o que demonstra que tal gestão se alinha ao planejamento das organizações.

O objetivo do planejamento é, de acordo com Andion y Fava (2002, p. 36), "fornecer aos gestores e suas equipes uma ferramenta que os municie de informações para a tomada de decisão, ajudando-os a atuar de forma proativa, antecipando-se às mudanças que ocorrem".

No contexto organizacional, o planejamento, segundo Estrada y Almeida (2007), pode ser entendido como um processo de tomada de decisões pré-estabelecidas e interdependentes, no qual a organização busca alcançar o futuro desejado.

Diante de um ambiente dinâmico, as organizações passam a se interessar por um planejamento que seja estratégico, que lhes permite enfrentar as ameaças, bem como aproveitar as oportunidades, oriundas deste 
ambiente. Para Araújo y Gonçalves (2011), o PE ajuda as organizações a se programarem, de modo a evitar surpresas onerosas comuns em tais ambientes.

O PE, para Fischmann y Almeida (2009, p. 27), é "uma técnica administrativa que, através da análise do ambiente de uma organização, cria a consistência das suas oportunidades e ameaças do ambiente de uma organização". Ansoff (1977), um dos primeiros estudiosos acerca de PE, o define como um processo sistemático e contínuo de desenvolvimento de estratégias que permite tomar decisóes sistemáticas e racionais, para que os resultados alcançados possam ser comparados com o planejado.

Para Estrada y Almeida (2007), o PE é uma técnica administrativa que visa ordenar as ideias dos sujeitos organizacionais, de modo a criar uma visão do caminho que deve ser seguido, além de possibilitar que as ações a serem realizadas para o alcance da visão de futuro esperada sejam ordenadas.

No contexto organizacional, a função do PE, para Fernandes et al. (2015, p. 5), é "descrever as condições internas de resposta ao ambiente externo e a forma de modificá-las visando o fortalecimento da instituição".

O PE, de acordo com Valente y De Macedo Brasil (2019), contribui para a compatibilização dos objetivos estratégicos organizacionais com a realidade do mercado, para que, assim, toda a organização se esforce para o cumprimento de seus propostos e para a perpetuação da organização no mercado em que atua.

A importância do PE para as organizações é destacada por Cruz et al. (2017) que afirmam que tal planejamento é indispensável para a obtenção dos resultados desejados e para aumentar a competitividade das organizações. Para os autores, o PE é questão de sobrevivência das organizações, o que faz com as organizações se voltem para o uso do planejamento como forma de potencializar seus resultados. Para sobreviverem e aumentarem a competitividade, as organizações devem se valer do conhecimento como recurso estratégico e, portanto, o mesmo precisa ser considerado no PE organizacional.

Para que os benefícios advindos do PE sejam aproveitados, as organizações devem fazer uso de ferramentas administrativas que, segundo Almeida y Oliveira (2020), auxiliam no processo de seleção e implementação da estratégia. Ainda em relação a estas ferramentas, os autores supracitados destacam a matriz SWOT como procedimento básico para a implantação do PE. Para Teixeira y Alonso (2014), ferramentas como a análise SWOT $($ Strengths $=$ forças, Weaknesses $=$ fragilidades, Opportunities $=$ oportunidades, Threats $=$ ameaças $)$, auxiliam na identificação dos principais obstáculos que afetam os resultados e objetivos organizacionais.

Diante do contexto atualmente vivido pelas organizações, onde o capital intelectual de seus colaboradores tem adquirido papel destaque, o PE, de acordo com Terra (2005) deve fazer uso da GC porque esta gestão possui papel central para a competitividade organizacional, uma vez que, conforme Teixeira Filho (2000, p. 17), "o conhecimento tornou-se o fator econômico mais importante no ambiente competitivo das organizações”.

\section{GeStÃo DA CONHECIMENTO}

O conhecimento é a representação da união entre experiências, valores, insights e informações contextuais e que tem sua origem na mente dos sujeitos, portanto, não costuma estar inserido apenas em sua forma explícita dentro dos documentos e repositórios, mas também na sua forma tácita e prática, advinda das pessoas que conhecem. Portanto, no contexto organizacional, o conhecimento está presente também nas rotinas, processos e práticas organizacionais, que são necessárias para o alcance de bons resultados corporativos, segundo Davenport y Prusak (1998).

As organizações que utilizam o conhecimento de modo adequado, de acordo com Bukowitz y Williams (2002), tem a possibilidade de aproveitar dos seguintes benefícios: desenvolvimento de processos eficientes e efetivos, geração de impactos positivos nos resultados financeiros por meio da redução de custos, redução do tempo dos ciclos (que faz com que se obtenha mais rapidamente o que necessita), entrega mais rápida do que o cliente necessita, incentivo a inovação e geração de mais valor ao cliente 
O conhecimento, conforme (Silva, 2007, p. 86), "é fonte para se obter vantagem competitiva, visto que alimenta os processos de gestão que planejam e implementam as estratégias de sobrevivência”, portanto, a gestão deste recurso é basilar para o PE das organizações.

O conhecimento, segundo Nonaka y Takeuchi (1997), pode ser tácito ou explicito. O conhecimento tácito, ainda de acordo com os autores, envolve o conhecimento pessoal, voltado a um contexto específico, difícil de ser formulado e comunicado, difícil de ser explicado e visualizado porque está enraizado nas ações, nas experiências, nos ideais, nos valores e nas emoções dos indivíduos. Já o conhecimento explícito é o conhecimento codificado, facilmente transmitido por linguagem formal e sistemática, que pode ser expresso em palavras, números ou sons, e compartilhado na forma de dados, fórmulas científicas, recursos visuais, fitas de áudio, especificações de produtos ou manuais (Nonaka y Takeuchi, 1997).

A conversão do conhecimento tácito em explicito e do explicito em tácito leva a criação do conhecimento organizacional. Os modos de conversão do conhecimento são representados pela "Espiral do conhecimento" ou "Modelo SECI" de Nonaka y Takeuchi (1997), que descreve como os conhecimentos tácito e explícito são amplificados em termos de qualidade e quantidade, assim como do indivíduo para o grupo e, então, para o nível organizacional. Nesta espiral, a criação do conhecimento se inicia com a socialização e passa por todos os modos de conversão do conhecimento, quais sejam: 1) Socialização: conversão de conhecimento tácito em tácito, processo de compartilhamento de experiências e habilidades individuais; 2) Externalização: conversão do conhecimento tácito em explícito, por meio de agrupamento e decodificação de metáforas, conceitos, analogias, modelos e hipóteses; 3) Combinação: conversão do conhecimento explícito em explícito que se dá pela sistematização de conceitos em um sistema de conhecimento e pelo emparelhamento de diferentes modos do conhecimento explícito; e 4) Internalização: conversão de conhecimento explícito em tácito, que envolve o "aprender fazendo".

Para que as organizações possam ampliar seus conhecimentos organizacionais, a gestão sobre tal recurso se faz essencial. A GC, de acordo com Teixeira Filho (2000, p. 22) é "uma coleção de processos que governa a criação, disseminação e utilização do conhecimento para atingir plenamente os objetivos da organização”. Para Dalkir (2005), a GC é a administração deliberada e sistemática de pessoas, tecnologias, processos e estrutura de uma organização, que tem por finalidade agregar valor à organização, por meio da reutilização do conhecimento e da inovação.

De acordo com Damian y Moro-Cabero (2020b), a GC em sua aplicação, é capaz de evitar a amnesia corporativa que pode ser prejudicial para as tomadas de decisão no âmbito das organizações. Além disso, pode ser entendida como um conjunto de processos e técnicas gerenciais voltadas à geração de valor, criação, manejo, disseminação e reutilização do conhecimento tácito e explícito, individual, grupal e organizacional.

Diretamente associada às práticas gerenciais e as etapas do aprendizado individual e compartilhado, a GC envolve a destinação de recursos e a utilização de técnicas gerenciais para gerar, disseminar e administrar os conhecimentos estratégicos, com a finalidade de gerar resultados econômicos satisfatórios (Terra, 2005). Por meio da definição deste autor, é possível verificar similitudes que contribuem para a utilização da GC junto ao PE, já que ambos se preocupam com recursos e técnicas organizacionais para o alcance dos resultados almejados. A GC, ainda de acordo com o autor, envolve, entre outras coisas, a análise das características e demandas do ambiente competitivo, o que, mais uma vez, reforça o uso de tal gestão junto ao planejamento organizacional.

A GC, segundo Santos y Damian (2018), pode ser definida como uma ferramenta que visa cuidar do conhecimento oriundo das pessoas, e dentro de uma organização, ela objetiva oferecer condições de aprendizagem, acesso e internalização de informações que serão posteriormente transformadas em conhecimento de modo natural e individual de cada pessoa (Santos y Damian, 2018).

Os principais benefícios da GC, que de acordo com Hoffmann (2009), são a aquisição de melhores desempenhos organizacionais; o desenvolvimento de produtos, serviços e sistemas informacionais são otimizados e possibilita evoluções expressivas no que diz respeito à melhoria da qualidade e à gestão de 
clientes. Para usufruir destes benefícios, as organizações precisam se dedicar a adequada implantação da GC em contexto organizacional, o que, para Del-Massa, Damian y Valentim (2018), é resultado de uma gestão organizacional eficiente, cujos benefícios ultrapassam os resultados imediatos ao proporcionar qualidade, criatividade e inovação de modo contínuo.

O crescente aumento das organizações pela GC é devido a globalização dos negócios; ao fato das organizações terem se tornado mais enxutas; a "amnesia corporativa" causada pela alta taxa de mobilidade da força de trabalho que dificulta a criação e a manutenção do conhecimento organizacional; e aos avanços tecnológicos (Dalkir, 2005).

A GC e o PE são para Michelon, Pilatti, Lima \& Carvalho (2006), meios que levam as organizações a se destacarem em ambientes altamente competitivos. Para os autores, como base competitiva, a utilização do conhecimento agrega valor a produtos e serviços, e o PE leva a resultados superiores quando utilizado para disseminação das diretrizes estratégicas e de feedback.

\section{Procedimentos metodológicos}

Foi realizada uma revisão bibliográfica acerca dos conceitos envolvidos no estudo, quais sejam PE e GC. A busca se deu por artigos, livros, teses e dissertações, já que, de acordo com Marconi y Lakatos (2013), esta revisão permite a consulta em diferentes fontes de informação e, assim, possibilita a reflexão sobre diferentes pesquisas em distintas fontes de informação. A revisão bibliográfica, segundo Fonseca (2002), possibilita ao pesquisador conhecer o que já foi desenvolvido sobre o assunto pesquisado.

Após a revisão bibliográfica, foi realizado um estudo de caso sobre o $\mathrm{PE}$ do ObsCoop, pois, de acordo com Yin (2001), os estudos de caso são indicados para situações em que os pesquisadores não tem muito controle sobre os eventos e quando o foco se concentra em fenômenos contemporâneos inseridos em algum contexto da vida real, como é o caso aqui estudado. O estudo de caso é o esforço de pesquisa mais indicado para a compreensão de fenômenos sociais complexos, sejam eles individuais, organizacionais, sociais e políticos, o que, mais uma vez, vai ao encontro das características do contexto estudado.

O estudo de caso, ainda de acordo com o autor supracitado, é indicado para o estudo de eventos contemporâneos, em situações nas quais os comportamentos relevantes não podem ser manipulados, mas onde é possível fazer observações diretas e entrevistas sistemáticas.

Para Eisenhardt (1989), o estudo de caso é voltado para o entendimento das dinâmicas presentes em um determinado cenário, o que vai ao encontro do proposto por este estudo que é verificar as contribuições da GC para o PE desenvolvido pelo Observatório de Cooperativas de Crédito em contexto virtual devido as limitações impostas pela pandemia do COVID-19.

Para a realização do estudo de caso, foi utlizada a observação participante.

\section{ANÁlise dos DADOs E RESUltados}

O ObsCoop da Faculdade de Economia, Administração e Contabilidade de Ribeirão Preto da Universidade de São Paulo (FEA-RP/USP) se constitui de uma rede de pesquisadores com a missão de produzir conhecimento relevante sobre as cooperativas agropecuárias e financeiras. Por meio de uma equipe multidisciplinar, o ObsCoop construiu um robusto e atualizado banco de dados sobre estas cooperativas, o que permite o desenvolvimento de importantes produtos e serviços de informação voltados a comunidade interessada nestas cooperativas, quais sejam: cooperados; gestores, pesquisadores, entidades de representação; formuladores de política pública; e agentes relevantes para o desenvolvimento econômico e social.

Com o objetivo de reforçar sua posição de referência no debate acadêmico e garantir a troca de conhecimento sobre as cooperativas brasileiras, o ObsCoop promove, em intervalos regulares de 
tempo, debates entre acadêmicos e profissionais atuantes em cooperativas e em organizações diretamente relacionadas as cooperativas. O ObsCoop ainda disponibiliza informações sobre pesquisadores, cursos e entidades de ensino e pesquisa que promovem conhecimento sobre cooperativismo e, com o intuito de proporcionar conhecimento e formação aos diversos elos da cadeia cooperativa, desenvolve cursos de especialização acerca do cooperativismo.

Para o desenvolvimento do seu PE, o ObsCoop contou com uma equipe formada por três pesquisadores de cooperativas, um pesquisador em gestão e em cooperativas, um pesquisador em GC e um profissional com destacada atuação na área de cooperativas. Durante o segundo semestre de 2020, esta equipe se reuniu todas as segundas-feiras, no período da manhã. Devido às limitações impostas pela pandemia, os encontros se deram de modo virtual, o que contribuiu para o desenvolvimento do PE do ObsCoop sem dispêndios de recursos financeiros, o que se apresenta, em muitos casos, como um dos grandes limitadores de tal desenvolvimento.

A formação da equipe seguiu as orientações da GC no que diz respeito a sua formação multidisciplinar. Além desta característica, outras podem ser citadas e relacionadas à GC como a descentralização do poder, a participação de todos e a delegação de tarefas.

Em um primeiro momento, houve a apresentação do ObsCoop, bem como de seus produtos e serviços à equipe como forma de equalizar o conhecimento de todos acerca do ObsCoop. Neste momento, assim como em muitos outros, foi possível verificar a importância do compartilhamento do conhecimento para o sucesso do desenvolvimento de seu PE.

$\mathrm{Na}$ sequência, se discutiu sobre os passos a seguir e quais ferramentas utilizar, iniciando pelas seguintes análises: 1) do ambiente geral do ponto de vista econômico, social, político, legal e tecnológico; 2) do ambiente operacional a partir dos elementos clientes, mão-de-obra, fornecedor e internacionalização; e 3) do ambiente interno relacionada aos aspectos pessoais, de marketing, de produtos e financeiros. Após a conclusão destas análises, foi possível definir a missão, a visão e os valores do ObsCoop. Neste momento, se faz oportuno ressaltar a importância do conhecimento e da experiência de um dos pesquisadores que participa do ObsCoop desde sua criação e que, atualmente, é o responsável pelo mesmo, demonstrando o importante aspecto destacado pela GC da importância do compartilhamento de experiências para a criação de novos conhecimentos. Um elemento essencial para a aquisição do conhecimento tácito é a experiência, por meio do compartilhamento da linguagem, observação, imitação e prática (Nonaka y Takeuchi, 1997).

Conforme destacado no referencial teórico, a matriz SWOT foi utilizada para identificação dos pontos fortes, pontos fracos, ameaças e oportunidades. Como tal matriz envolve aspectos internos e externos, tanto a participação de membros do ObsCoop como de membros externos foi fundamental. Durante o levantamento dos elementos constituintes da matriz, mais uma vez, a participação de uma equipe multidisciplinar, com diferentes níveis de conhecimentos em áreas complementares, bem como experiências distintas, contribuiu de maneira basilar para o resultado alcançado.

Para a realização do PE é preciso que as organizações disponham de recursos adequados, sobre os quais Barney (1991) destaca os recursos internos, uma vez que as fontes de vantagens competitivas sustentáveis são baseadas em recursos internos valiosos, raros e difíceis de imitar, como é o caso dos recursos humanos. Neste sentido, Silva (2004) afirma que as organizações devem investir em GC para criar vantagem competitiva sustentável e de difícil imitação, já que está enraizada nos sujeitos organizacionais e não em recursos físicos que podem ser facilmente imitáveis. Seguindo estas recomendações, foi observado que os membros da equipe foram os elementos mais valorizados no processo do PE do ObsCoop, o que vai ao encontro de Damian y Moro-Cabero (2020c, p. 1) que afirmam que "As pessoas são consideradas um importante viabilizador da GC porque são elas que captam, criam, armazenam, compartilham e aplicam conhecimento.”

Valente y De Macedo Brasil (2019) afirmam que o PE deve ser alinhado com os objetivos organizacionais. O mesmo ocorre em relação a GC, quando Batista (2012) traz que é fundamental alinhar a GC com os objetivos estratégicos e as metas, de modo que a GC seja um instrumento para alcançar os resultados 
organizacionais. Estas orientações foram seguidas durante o desenvolvimento do PE do ObsCoop, já que houve a preocupação constante de alinhar o PE e a GC aos objetivos do ObsCoop.

As organizações precisam criar condições adequadas para que a conversão do conhecimento e a consequente criação do conhecimento organizacional se deem de forma efetiva. Essas condições, que devem ser especificas para cada modo de conversão, foram sugeridas por Silva (2004) e utilizadas no desenvolvimento do PE do ObsCoop:

- Na socialização, o conhecimento foi compartilhado por meio de diálogos frequentes que ocorriam durante as reuniões semanais e também por trocas de e-mails e mensagens no WhatsApp; a comunicação face a face foi possível graças ao uso de tecnologias de informação e comunicação, brainstorming era recurso frequente nas reuniões semanais, assim como o compartilhamento de experiências e o trabalho em equipe.

- Na externalização, houve a transmissão do conhecimento por meio de representações simbólicas do conhecimento tácito através de modelos e conceitos construídos por meio do uso de tecnologias que permitem a construção de documentos compartilhados.

- Na combinação, houve a conversão do conhecimento por meio da criação de documentos onde o conhecimento foi organizado e sumarizado.

- Na internalização, a conversão do conhecimento se deu através da leitura/visualização e do estudo individual de documentos de diferentes formatos/tipos (textos, imagens etc.) e da prática individual, que resultou, entre outras coisas, no desenvolvimento de trabalhos científicos.

De acordo com Takeuchi y Nonaka (2008), uma organização faz GC quando, entre outras coisas, cria espaços favoráveis à troca de experiências; admite erros; favorece intercâmbios entre seus colaboradores; reconhece e valoriza a diversidade; reconhece e valoriza seus talentos; busca sempre a inovação; dissemina a cultura da visão compartilhada; sistematiza e organiza o conhecimento adquirido; permite a redundância; reconhece no conflito um início de "caos criativo"; valoriza a gerência intermediária; incentiva a delegação e a autonomia; investe em capacitação; propicia elementos para a visão sistêmica; utiliza a tecnologia como uma ferramenta de apoio; possibilita acessos às informações em todos os níveis; incentiva a criatividade; e propicia ambientes lúdicos.

Em relação a afirmação acima, por meio da observação participativa, tem-se que:

- Os espaços virtuais, onde as reuniões foram realizadas, se mostraram altamente favoráveis à troca de experiências. As reuniões se davam em um ambiente rico de trocas e discussóes por parte de todos os participantes, sem distinção de níveis hierárquicos ou qualquer forma de poder.

- Visões equivocadas ou divergentes foram tratadas de forma pacifica, com muito respeito e sempre traziam aportes importantes ao trabalho desenvolvido.

- Houve um intenso intercâmbio de informações, conhecimento e experiências entre todos os membros da equipe.

- A diversidade sempre foi muito reconhecida porque era evidente o quanto agregava de conhecimento aos participantes e ao desenvolvimento de um PE adequado ao ObsCoop.

- Os talentos foram reconhecidos e valorizados por meio de oportunidades de novos trabalhos que surgirão em virtude deste planejamento, além da delegação e autonomia que representam fatores motivacionais importantes.

- A inovação foi a base para avaliação dos elementos da Matriz SWOT e, a partir daí, para o desenvolvimento de produtos e serviços que serão disponibilizados pelo ObsCoop.

- Todas as etapas do PE se deram meio a cultura da visão compartilhada, com a participação e consenso de todos, o que levou, em alguns momentos, ao "caos criativo". 
- Todo conhecimento adquirido no desenvolvimento deste planejamento foi sistematizado e organizado para permitir seu uso para o desenvolvimento dos próximos passos do ObsCoop.

- Para o desenvolvimento do PE foi necessário equalizar o conhecimento de todos quanto ao ObsCoop, o que fez com que todos tivessem uma visão sistemática acerca da organização e de seu ambiente.

- A tecnologia foi de vital importância, uma vez que todos os encontros se deram de modo virtual e para estimular a participação de todos, documentos colaborativos foram utilizados.

- Para o desenvolvimento do PE, todos os membros da equipe tiveram acesso às informações em todos os níveis.

- A intensa troca de informações e conhecimento, aliada a um ambiente participativo, levou ao desenvolvimento de importantes inovações em produtos, serviços e processos destacados no PE.

Ações como permitir a redundância, valorizar a gerência intermediária, investir em capacitação e propiciar ambientes lúdicos nas suas atividades rotineiras, não foram destacadas porque ficam restritas quando trazidas ao contexto especifico do desenvolvimento do PE em modo virtual e por características do próprio ObsCoop, como o fato de ser uma organização de pequeno porte. Se ressal ta, por exemplo, que o ObsCoop investe muito em capacitação por meio da participação em pesquisas cientificas, treinamentos, participação em palestras, seminários e congressos, porém tal aspecto fica limitado na esfera tratada por este estudo.

\section{Conclusões}

O PE tem sido cada vez mais utilizado pelas organizações por sua eficácia na análise de ambientes internos e externos, e no fornecimento de informações estratégicas para processos decisórios, onde são considerados os pontos fortes, os pontos fracos, as oportunidades e as ameaças que compõem o contexto organizacional, de modo que as organizações tenham condições de definir e atingir metas e objetivos estratégicos factíveis com os recursos que dispõem.

Dentre os recursos utilizados pelas organizações para atingirem seus objetivos estratégicos, o papel de destaque tem sido ocupado pelo conhecimento que, devido as suas características únicas, auxilia diretamente na construção de vantagem competitiva sustentável, o que justifica o interesse das organizações por sua gestão.

Como auxiliam diretamente na tomada de decisão, na inovação, na utilização de recursos, no desenvolvimento de produtos e serviços, entre outros, os benefícios advindos do PE e da GC se fazem ainda mais evidentes em momentos difíceis, como, por exemplo, na situação atual vivenciada pela pandemia do COVID-19. Foi neste cenário que o ObsCoop, por meio do uso de tecnologias de informação e comunicação e contando com uma equipe multidisciplinar de especialistas, desenvolveu seu PE. Para alcançar o objetivo proposto, as contribuições advindas da GC foram essenciais.

A construção de um ambiente colaborativo, onde as experiências e conhecimentos dos integrantes da equipe eram compartilhados, a descentralização, o trabalho em equipe, a delegação, a autonomia e a motivação, foram algumas contribuições aportadas pela GC que possibilitaram que o resultado almejado fosse alcançado.

Os recursos organizacionais, com destaque aos recursos financeiros, se fazem ainda mais escassos em um cenário de pandemia. Porém, conforme demonstrado pelo estudo de caso apresentado, a escassez de recursos financeiros não foi um fator impeditivo para o desenvolvimento do PE que contou com as contribuições da GC. Diante do estudo de caso apresentado, é possível concluir que as contribuições da GC foram fundamentais para o desenvolvimento do PE do ObsCoop em meio a pandemia do COVID-19.

Por meio do estudo de caso realizado, é possível concluir que a GC contribui de modo efetivo para o PE e para o atingimento dos objetivos organizacionais, porquanto deve ser considerado como ferramenta basilar para o desenvolvimento e implantação de tal planejamento. 


\section{REFERÊNCIAS}

Alday, H. E. C. (2000). O planejamento estratégico dentro do conceito de administração estratégica. Revista da FAE, 3(2), 9-16. Recuperado de https://revistafae.fae.edu/revistafae/article/view/505

Almeida, J. S. C., \& Oliveira, S. S. (2020). Planejamento Estratégico em Empresas de Pequeno Porte. Revista Gestão \& Sustentabilidade, 2(1), 38-56. Recuperado de https://periodicos.uffs.edu.br/index.php/RGES/article/view/ 9780

Almeida, M. I. R. de. (2010). Manual de planejamento estratégico: desenvolvimento de um plano estratégico com a utilização de planilhas excel. 3. ed. São Paulo: Atlas.

Andion, M. C. \& Fava, R. (2002). Gestão empresarial. Curitiba: Associação Franciscana de Ensino Bom Jesus.

Ansoff, I. (1977). Estratégia empresarial. São Paulo: McGraw-Hill.

Araújo, F. De., \& Gonçalves, C. A. (2011). O processo de formulação e implementação de planejamento estratégico em instituições do setor público. Revista de administração da Universidade Federal de Santa Maria, 4(3), 458-476. Recuperado de https://www.redalyc.org/pdf/2734/273421614010.pdf

Barney, J. B. (1991). Firm resources and sustained competitive advantage. Journal of Management, 17(1), 99-120. Recuperado de https://journals.sagepub.com/doi/10.1177/014920639101700108

Batista, F. F. (2012). Modelo de gestão do conhecimento para a administração pública brasileira: como implementar a gestão do conhecimento para produzir resultados em benefício do cidadão. Brasília: Ipea.

Bukowitz, W.R., \& Williams, R.L. (2002). Manual de gestão do conhecimento: ferramentas e técnicas que criam valor para a empresa. Porto Alegre: Bookman.

Chiavenato, I. (2014). Introdução a teoria geral da administração. São Paulo: Manole.

Cruz, D. M. de B.; Neris, L. M.; Vilas-Boas, L. G. \& Menezes, J. D.de. S. (2017). Aplicação do planejamento estratégico a partir da análise SWOT: Um estudo numa empresa de tecnologia da informação. In: Anais do 9 SIMPROD. Universidade Federal de Sergipe, Aracajú. Recuperado de https://mail.fsssacramento.br/arquivos/epo/iniciaca o-cientifica/artigo-1.pdf

Dalkir, K. (2005). Knowledge management in theory and practice. Linacre House, Jordan Hill, Oxford: Elsevier.

Damian, I. P. M. \& Moro-Cabero, M. M. M. (2020a). Proposição de um modelo de gestão do conhecimento voltado às características da memória organizacional. Encontros Bibli: revista eletrônica de biblioteconomia e ciência da informação, 25, 01-21. Recuperado de https://periodicos.ufsc.br/index.php/eb/article/view/1518-2924.2020. e73691/44028

Damian, I. P. M. \& Moro-Cabero, M. M. (2020b). Inter-relações entre gestão do conhecimento e memória organizacional. Palabra clave, 10(1), e106. Recuperado de https://www.palabraclave.fahce.unlp.edu.ar/article/ view/PCe106/12701

Damian, I. P. M. \& Moro-Cabero, M. M. (2020c). Diretrizes estratégicas baseadas nos fatores Críticos de sucesso da gestão do conhecimento voltadas às características da memória organizacional. Informação \& sociedade: estudos, 30(2), 1-25. Recuperado de https://periodicos.ufpb.br/ojs2/index.php/ies/article/view/52478

Davenport, T. H. \& Prusak, L. (1998). Conhecimento empresarial. Rio de Janeiro: Campus.

Del-Massa, H. C. O.; Damian, I. P. M. \& Valentim, M. L. P. (2018). Competência em informação no apoio à gestão do conhecimento. Informação \& sociedade. estudos, 28(1). Recuperado de https://knowledgesociety.usal.es/syst em/files/CoInfo\%20no\%20apoio\%20a\%20GC.pdf

Eisenhardt, K. M. (1989). Building theories from case study research. The Academy of Management Review, 14(4), 532-550. Recuperado de https://www.jstor.org/stable/258557?seq=1\#metadata_info_tab_content

Estrada, R. J. S. \& De Almeida, M. I. R. (2007). A eficiência e a eficácia da gestão estratégica: Do planejamento estratégico à mudança organizacional. Ciências da administração, 9(19), 147-178. Recuperado de https://perio dicos.ufsc.br/index.php/adm/article/view/1784

Fernandes, I. G. M. et al. (2015). Planejamento estratégico: análise SWOT. Revista conexão eletrônica das Faculdades Integradas de Três Lagoas, 8(1). Recuperado de https://bit.ly/3rmtxEY 
Fischmann, A. A. \& Almeida, M. I. R. (2009). Planejamento estratégico na prática. São Paulo: Editora Atlas.

Fonseca, J. J. S. (2002). Metodologia da pesquisa cientifica. Fortaleza: UEC.

Fresneda, P. S. V.; Gonçalves, S. M. G.; Papa, M. \& Fonseca, A. F. (2009). Diagnóstico da gestão do conhecimento nas organizaçôes públicas utilizando o método organizational knowledge assessment (OKA). En II Congresso Consad de Gestão Pública, Brasilia, Brasil. Recuperado de http://consad.org.br/wp-content/uploads/2013/02/DIAGN\%C3\%93STICO-DA-GEST\%C3\%83O-D O-CONHECIMENTO-NAS-ORGANIZA\%C3\%87\%C3\%95ES-P\%C3\%9ABLICAS-UTILIZANDO-OM\%C3\%89TODO-ORGANIZATIONAL-KNOWLEDGE-ASSESSMENT-OKA2.pdf

Hoffman, W. A. M. (2009). Gestão do conhecimento: desafios de aprender. São Carlos: Compacta.

Marconi, M. A. \& Lakatos, E. M. (2013). Técnicas de pesquisa: planejamento e execução de pesquisas, amostragens e técnicas de pesquisa, elaboração, análise e interpretação dos dados. São Paulo: Atlas.

Michelon, M. J.; Pilatti, L. A.; Lima, I. A. de \& Carvalho, H. G. de. (2006). A criação do conhecimento corporativo promovido pelos fluxos de informações gerados na implantação do planejamento estratégico. Revista produção online, 6(1).

Nonaka, I. \& Takeuchi, H. (1997). Criação de conhecimento na empresa: como as empresas japonesas geram a dinâmica da inovação. Rio de Janeiro: Elsevier.

Santos, B. R. P. \& Damian, I. P. M. (2018). O mapeamento do conhecimento por meio da análise SWOT: estudo em uma organização pública de saúde. Em questão, 24, 253-274. Recuperado de https://seer.ufrgs.br/EmQuestao/ article/view/76910/0

Silva, S. L. (2004). Gestão do conhecimento: uma revisão crítica orientada pela abordagem da criação do conhecimento. Ciência da informação, 33(2), 143-151. Recuperado de https://www.scielo.br/pdf/ci/v33n2/a15v33n2.pdf

Silva, H. M. da. (2007). Gestão do conhecimento e inteligência competitiva em organizações: uma abordagem conceitual. Revista de iniciação vientifica da FFC, 7(1), 84-93.

Takeuchi, H. \& Nonaka, I. (2008). Gestão do conhecimento. Porto Alegre: Bookman.

Teixeira, C. A. \& Alonso, V. L. C. (2014). A Importância do Planejamento Estratégico para as Pequenas Empresas. En Anais do 11 Simpósio de excelência em gestão e tecnologia (SEGeT). Rio de Janeiro, Brasil. Recuperado de htt ps://www.aedb.br/seget/arquivos/artigos14/132020.pdf

Teixeira filho, J. (2000). Gerenciando conhecimento: como a empresa pode usar a memória organizacional e a inteligência competitiva no desenvolvimento de negócios. Rio de Janeiro: SENAC.

Terra, J. C. C. (2005). Gestão do conhecimento: o grande desafio empresarial. São Paulo: Negócio Editora.

Valente, D. L. \& De Macedo Brasil, C. V. (2019). Análise de ferramentas administrativas para o planejamento estratégico. FESPPR pública, 3(1), 1-13. Recuperado de http://publica.fesppr.br/index.php/publica/article/vi ew/162

Wong, K. Y. \& Aspinwaal, E. (2004). Knowledge management implementation frameworks: a review. Knowledge and process management, 11(2), 93-104. https://doi.org/10.1002/kpm.193

Yin, R. K. (2001). Estudo de caso: planejamento e métodos. Porto Alegre: Bookman. 